\title{
Structure, charge density and energetic features of quinoline derivatives
}

Magdalena Woinska ${ }^{1}$, Monika Nowakowska ${ }^{1}$, Przemyslaw Taciak ${ }^{2}$, Paulina M. Dominiak ${ }^{1}$, Krzysztof Wozniak $^{1}$

${ }^{1}$ Faculty Of Chemistry, University Of Warsaw, Warsaw, Poland, ${ }^{2}$ Faculty of Pharmacy, Medical University of Warsaw, Warsaw, Poland

E-mail: mwoinska@chem.uw.edu.pl

Quinoline derivatives are common among many natural compounds; moreover, quinoline is a structural subunit which plays an important role in the design and synthesis of pharmaceutically active substances. This makes investigation of its derivatives and their properties particularly interesting and is the motivation for the presented $X$-ray diffraction and computational study of crystal structure, electron density (ED) and intermolecular interactions of four quinoline derivatives. The derivatives differ in the substituent at position 8 which is either a halogen atom ( $\mathrm{Cl}, \mathrm{Br}, \mathrm{I})$ or -S-Ph moiety. Highresolution X-ray measurements were performed in each case, resulting in experimental data of varying quality, possibly characterized by the presence of anharmonic motion, which makes them suitable for verifying capabilities of two different charge density models compared in this work.

The refinement methods applied during X-ray data processing were: the multipole refinement (MR) in Hansen and Coppens formalism [1] and the X-ray wavefunction refinement (XWR) [2] implementing Hirshfeld partition of ED. The latter technique includes two steps: (a) Hirshfeld atom refinement (HAR) being only structural refinement and (b) X-ray constrained wavefunction fitting introducing experimental contribution to ED. According to the performed estimate, data resolution was not always sufficient to refine the 3rd and 4th order of anharmonicity of $\mathrm{Cl}, \mathrm{Br}, \mathrm{I}$ and $\mathrm{S}$ atoms in the investigated structures. Nevertheless, an attempt of such refinement was made in all the cases to check if the tested refinement techniques tended to detect the non-existing effect when additional parameters were introduced into the model. The analysis of refinement of various orders of anharmonicity and the dependence of the results on data quality and the model used will be presented. Proper establishing of hydrogen positions and treatment of its thermal motion is necessary for correct description of ED and related properties. XWR allowed refinement of hydrogen positions and ADPs in the considered compounds, however precision and accuracy was strongly dependent on data quality. With MR it was possible to freely refine hydrogen ADPs only for good-quality data sets, while refinement of hydrogen positions was not successful and restraining them to mean neutron distances [3] was necessary. Moreover, the experimental ED was subject to analysis in order to compare the ability of MR and XWR to model density features and evaluate performance of the methods in the case of data with evident presence of experimental errors. Periodic DFT calculations were performed to serve as a benchmark for the experimental results.

Additionally, interactions in the crystal lattice were studied. The $\mathrm{Cl}$-, $\mathrm{Br}$ - and $\mathrm{Ph}-\mathrm{S}$-substituted derivatives are characterized by a 3-dimensional network formed by contacts such as $\mathrm{N}-\mathrm{H} \ldots \mathrm{O}, \mathrm{C}-\mathrm{H}$...S/Cl/Br or $\mathrm{C}-\mathrm{H}$...O. Crystal lattice of the iodinecontaining compound consists of 2-dimensional layers mutually linked by pi-stacking interactions. The molecules within layers are connected by $\mathrm{C}-\mathrm{H} \ldots \mathrm{O}$ and $\mathrm{N}-\mathrm{H}$.... I hydrogen bonds, as well as $\mathrm{C}-\mathrm{I}$...O halogen bonds. Calculations of cohesive energy, molecule deformation energy, energy of interlayer interactions and dimer interaction energies were performed for the experimental and theoretical geometries of the investigated structures.

Polish National Science Centre MAESTRO grant decision number DEC2012/04/A/ST5/00609 is acknowled

[1] Hansen, N. K. \& Coppens, P. (1978). Acta Cryst. A34, 909-921.

[2] Grabowsky, S.; Luger, P.; Buschmann, J.; Schneider, T.; Schirmeister, T.; Sobolev, A. N. \& Jayatilaka, D. (2012). Angew. Chem. Int. Ed., 51, 6776-6779.

[3] Allen, F. H. \& Bruno, I. J. (2010). Acta Cryst. B, 66, 380-386. 


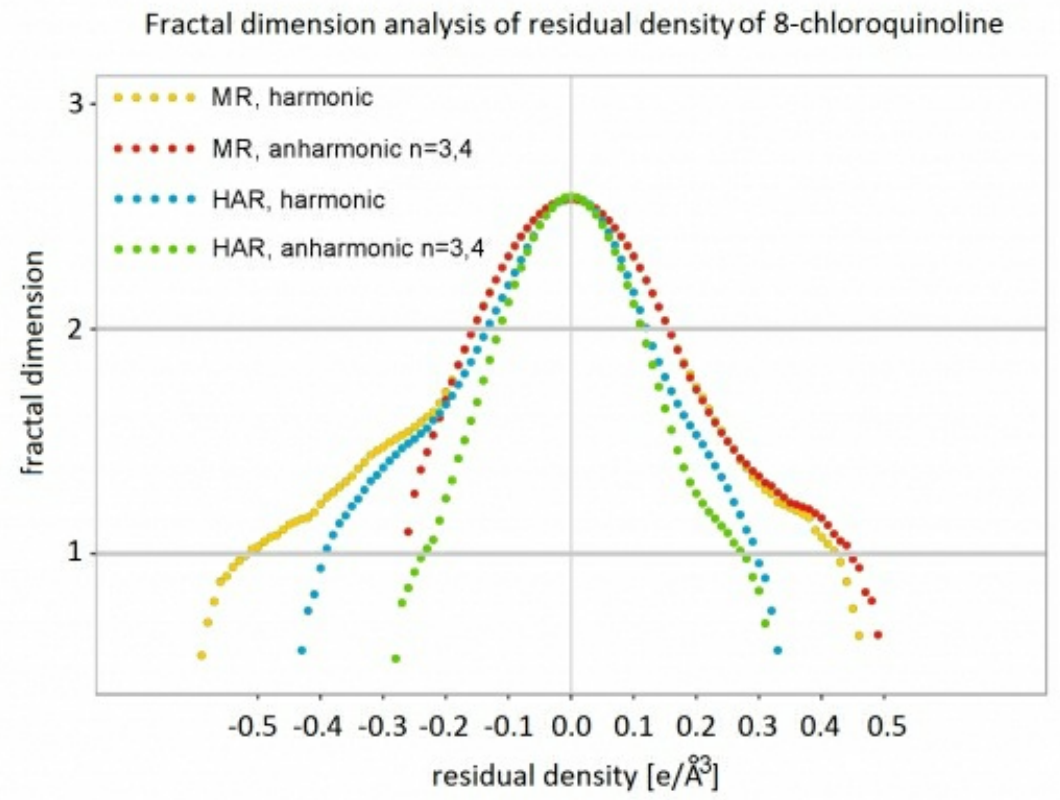

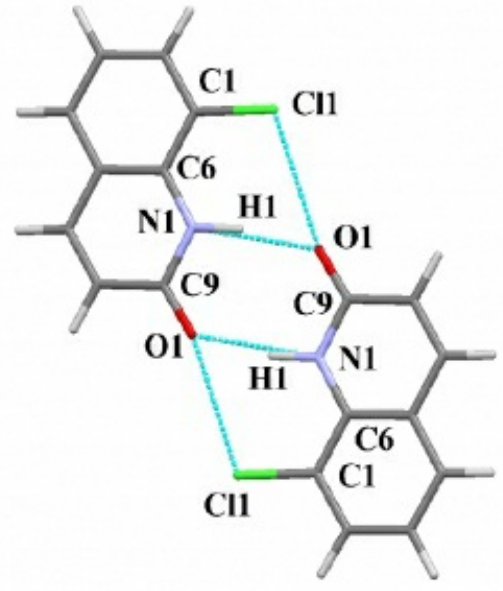

Dimer motif in the crystal lattice of 8-chloroquinoline

Keywords: $\underline{\text { X-ray wavefunction refinement, multipole refinement, quinoline derivatives }}$ 